\title{
The Effect of Virtual Haptic Training on Real Surgical Drilling Proficiency
}

\author{
Christopher Sewell $^{*} \quad$ Nikolas H. Blevins ${ }^{\dagger} \quad$ Sumanth Peddamatham ${ }^{\ddagger} \quad$ Hong Z. Tan ${ }^{\ddagger}$ \\ Dan Morris ${ }^{*}$ Kenneth Salisbury ${ }^{*}$ \\ (*) Department of Computer Science, Stanford University, Stanford, CA, USA \\ $(\dagger)$ Department of Otolaryngology, Stanford University, Stanford, CA, USA \\ (¥)Haptic Interface Research Laboratory, Purdue University, West Lafayette, IN, USA \\ E-mail: \{csewell,nblevins\}@stanford.edu, jks@robotics.stanford.edu,peddamat@gmail.com, \\ hongtan@purdue.edu,dmorris@cs.stanford.edu
}

\begin{abstract}
This sketch presents the design and preliminary results of a study that investigates the transference of motor skills learned in a haptic-enabled virtual environment to performance on a surgically-relevant task in the real world. The chosen task, which requires skills similar to those needed in a number of surgical procedures, including stapedotomy and cochleostomy, is drilling holes through an eggshell using a surgical drill without penetrating the egg's inner membrane. Results reflect a learning curve for task proficiency and indicate a benefit to haptic training, but a planned follow-up study will be required for this claim to be statistically conclusive.
\end{abstract}

\section{Introduction}

Since the inception of the field of computer haptics, researchers have been excited about the potential of applying this technology to train motor skills to better perform tasks in which force-feedback is an essential component. The growing field of surgical simulation, in particular, has been increasingly incorporating haptic devices in hopes of improving their educational value.

Several studies, including those by Feygin et. al. [1] and by Morris et. al. [2], have shown that participants who have been taught a force trajectory using visuohaptic training in a simulator are better at recalling that trajectory within the virtual environment than those taught using only visual training. A smaller number of studies have demonstrated transference of skills from a haptic simulator to a simple real-world task, such as building a Lego model (Adams et. al. [3]) or hitting a ball in table tennis (Todorov et. al. [4]). Baillie et. al. [5] showed a drastic increase in veterinary students' ability to locate the uterus in cows after using a haptic simulator. Other studies have shown that participants trained on (non-haptic) surgical simulators are faster and make fewer errors in the operating room than those who have not received simulator training (as summarized by Haque et. al. [6]). Most notable among these have been Seymour et. al. [7] and Grantcharov et. al. [8] using the MIST-VR laparoscopic simulator, which does not include haptic feedback. However, little is yet known about the transference of specific motor skills learned in a haptic simulator to surgicallyrelevant tasks performed in the real world.

In this sketch, we present the experimental design and results of a preliminary study in which we explore the effect of training in a haptically-enabled virtual environment on performance of a surgically-relevant real-world task. The task we have chosen is drilling holes through the shell of a chicken egg with a surgical drill without piercing the underlying membrane. This was chosen due to the similarity of this task to a number of difficult and important surgical tasks in which a thin layer of bone must be removed without pushing through into an underlying structure when the bone finally gives way. One important procedure involving this task in ear surgery is a stapedotomy, in which the thin bone of the stapes footplate must be drilled without pushing through the underlying membrane into the saccule, causing severe inner ear injury. Anecdotal notes from surgeons liken this bone to the shell of a chicken egg in thickness and hardness.

\section{Force Measurements}

Drilling force was measured with an ATI nano-17 force/torque sensor with temperature compensation. The sensor was mounted under a clear dish that holds an egg with silly putty (Fig. 1). The dish and 
supporting plate are bolted together, and the egg hardly moved relative to the silly putty, so friction and viscosity issues are avoided with this set-up. Fig. 2 shows the force traces for drilling a hole on top of an egg. It is clear that the main component was along the $-z$ (downward) direction with a maximum force between 1 and 1.5 Newton. When drills with larger burrs were used, the maximum force could increase to 2-3 Newtons.

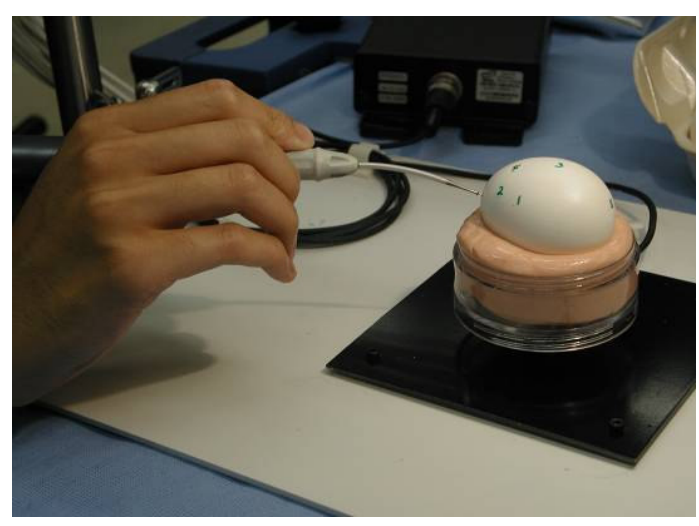

Figure 1. Set-up for force measurements.

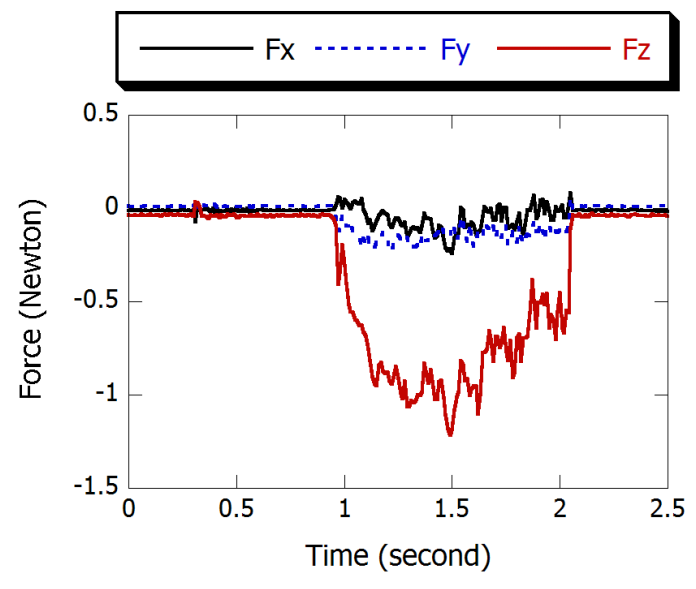

Figure 2. Recorded force measurements.

\section{Experimental Design}

A simulator was designed to isolate the training of a specific motor skill: minimizing the penetration distance of a drill when it pops through a thin layer of resistance. In the simulator, the user controls a virtual drill with a Phantom haptic device and applies a force downwards against a horizontal virtual plane (Fig. 3). Once a force greater than 0.3 Newtons (based on results obtained in Sec. 2) has been applied for a predetermined time (randomly chosen for each trial, between 8 and 12 seconds, roughly corresponding to the amount of time usually taken for this task in the operating room), the resistance of the plane suddenly disappears, and the user is scored according to the maximum distance the virtual drill falls downwards immediately after the plane's resistance disappears.

Ten participants were randomly assigned to two 5member groups. Both groups were shown a brief video showing an expert correctly drilling holes in an egg. Those in Group A were then asked to drill eight holes (at locations marked with black dots equally spaced around the circumference of the egg, a third of the way from the top) in each of four eggs (Fig. 4). The eggs were mounted in a holder, and a real surgical drill ( $3 \mathrm{~mm}$ diamond burr) was used. Subjects were instructed to make each hole at least as wide as the diameter of the drill burr at its widest point without puncturing the membrane underneath the shell. They were given 15 seconds to drill each hole and were provided with a five-second warning. The proctor would then evaluate each hole by lightly tapping the membrane with a pencil in order to determine whether it had been pierced, and by attempting to insert the drill, while turned off, all the way through the hole, in order to determine if it was wide enough. The subject was given feedback after drilling each hole. Participants in Group B performed twenty-four trials on the simulator, and then drilled eight holes in one egg using the aforementioned procedure.

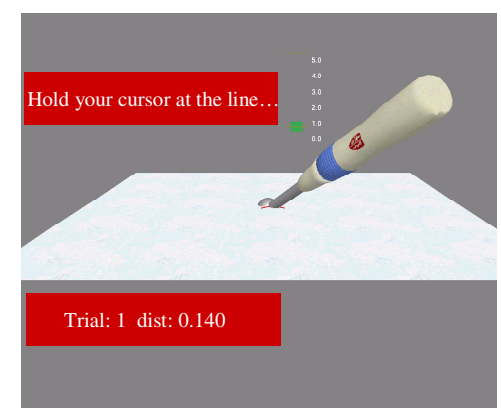

Figure 3. Screen-shot of simulator.

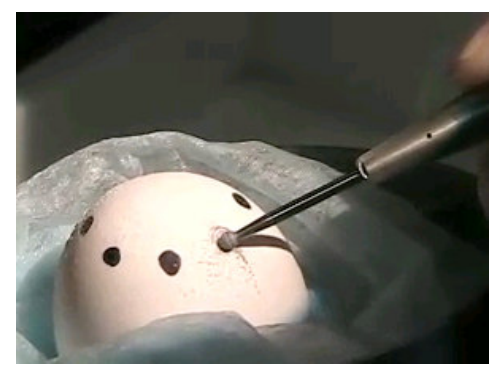

Figure 4. Set-up for drilling trials. 


\section{Results}

The numbers of correctly-drilled (sufficiently large hole and intact membrane) eggs for the two groups are shown in Fig. 5. A learning effect can be observed for Group A which correctly drilled, on an average, 2.6 of the eight holes in the first egg, 4.0 in the second egg, 5.2 in the third, and 5.4 in the fourth. Group B correctly drilled an average of 4.2 of the eight holes on their first egg. Thus, while Group B was not as proficient after 24 simulator trials as Group A was after drilling 24 holes in real eggs (i.e., comparing Group B's first real egg to Group A's fourth egg), they did tend to start out further along the learning curve once they began working with real eggs. Group B took an average of only 4 trials before correctly drilling a hole on a real egg, while Group A took an average of 7 trials. However, due to the relatively high variance and the limited number of participants in this preliminary study, the results were not statistically conclusive.

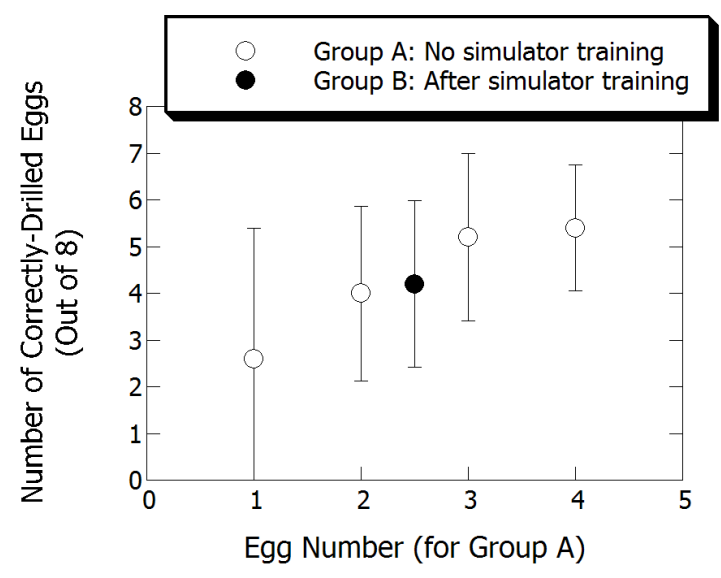

Figure 5. Experimental results.

\section{Discussion}

Assuming that the results presented in this sketch can be reproduced in a larger-scale study, we will show that a haptic virtual environment can train motor skills relevant to a surgical task such that trainees are more proficient when they begin performing the real task than if they had no such prior training. As our data suggest, a given number of simulator trials does not appear to be as effective as the same number of realworld trials, but, given the availability and costefficiency of using a simulator, many additional simulator trials could be completed. Future work will improve the simulator, recruit more participants, explore the learning curve for the haptic training group, employ additional experimental conditions to better separate the skill of preserving the membrane from that of making a sufficiently large hole, and record and analyze force profiles during egg drilling,

\section{Acknowledgments}

Support was provided by NIH LM07295. We would like to acknowledge KeonWoo Kim for his assistance with the experimental set-up, as well as Federico Barbagli and Sumit Agrawal for their input. We would also like to thank Medtronic Incorporated for allowing us the use of their otologic drills.

\section{References}

[1] D. Feygin, M. Keehner, and F. Tendick, "Haptic Guidance: Experimental Evaluation of a Haptic Training Method for a Perceptual Motor Skill”, 10th IEEE Haptics Symposium, March 2002.

[2] D. Morris, H.Z. Tan, F. Barbagli, T. Chang, and K. Salisbury, "Haptic Feedback Enhances Force Skill Learning", to appear in Proceedings of the 2007 World Haptics Conference, Tsukuba, Japan, Mar. 22-24, 2007.

[3] R.J. Adams, D. Klowden, and B. Hannaford, "Virtual Training for a Manual Assembly Task", Haptics-e, Vol. 2.2, October 2001 .

[4] E. Todorov, R. Shadmehr, and E. Bizzi, "Augmented Feedback Presented in a Virtual Environment Accelerates Learning of a Difficult Motor Task", Journal of Motor Behavior, Vol. 29, No. 2, pp. 147-158, 1997.

[5] S. Baillie, A. Crossan, S. Brewster, D. Mellor, and S. Reid, "Validation of a Bovine Rectal Palpation Simulator for Training Veterinary Students", Studies in Health Technology \& Informatics, 111:33-36, 2005.

[6] S. Haque and S. Srinivasan, "A Meta-Analysis of the Training Effectiveness of Virtual Reality Surgical Simulators", IEEE Transactions on Information Technology in Biomedicine, 10(1), pp. 51-58, January 2006.

[7] N.E. Seymour, A.G. Gallagher, S.A. Roman, M.K. O'Brien, V.K. Bansal, D.K. Andersen, and R.M. Satava, "Virtual Reality Training Improves Operating Room Performance", Annals of Surgery 2002, 236(4): 458464.

[8] T.P. Grantcharov, V.B. Kristiansen, J. Bendix, L. Bardram, J. Rosenberg, P. Funch-Jensen, "Randomized Clinical Trial of Virtual Reality Simulation for Laparoscopic Skills Training. British Journal of Surgery 2004, 91: 146-150. 